\title{
ĐCheck List
}

అ

\section{The Cossidae (Lepidoptera) of Mongolia}

\section{Roman Yakovlev}

Altai State University, South Siberian Botanical Garden, Lenina 61. RF-656049. Barnaul, Russia

Tomsk State University, Laboratory of Biodiversity and Ecology, Lenina pr. 36, 634050 Tomsk, Russia

E-mail: cossus_cossus@mail.ru

\begin{abstract}
An annotated list of the Cossidae of Mongolia includes two subfamilies, 12 genera, and 23 species.
\end{abstract}

Key words: species list, endemism, Palaearctic, Eurasia

\section{INTRODUCTION}

The Cossidae (Insecta, Lepidoptera), known as Carpenter and Leopard moths, are geographically widespread including approximately 1,000 species, 350 of which have been recorded from the Palearctic (Yakovlev 2011; Nieukerken et al. 2011).

The taxonomic knowledge of the Cossidae (Lepidoptera) of Mongolia was initiated with the collection of material during the expeditions of the wellknown Hungarian entomologist Zoltan Kaszab (Daniel 1965, 1969, 1970, 1973). In 2004, I published a synopsis of the Cossidae of Mongolia. Data on systematics and distribution of the Cossidae were summarized, treating fifteen species, including several new species, based mostly on material in the Thomas Witt Collection and material collected by myself in the Altai Mountains and southwestern (Dzhungarian Gobi) Mongolia (1999, 2002-2009). Finally, Yakovlev and Doroshkin (2004), Yakovlev (2006, 2007a, 2007b, 2009, 2011, 2012) described additional new species and provided distributional data on other species of Cossidae from Mongolia.

Additional collections of Cossidae during expeditions from 2010-2012 have added numerous new records requiring an update of the Cossidae of Mongolia.

\section{MATERIALS AND METHODS}

Adult moths were collected at night using ultraviolet lights. Male and female genitalia dissections were mounted in euparal on microscope slides. Taxonomic nomenclature used in this checklist follow Yakovlev (2011).

The abbreviations of collections, institutions, and museums include: ASL, collection of A. Saldaitis (Lithuania); LSL, Linnean Society (London, G.B.);
MHUB, Museum für Naturkunde der HumboldtUniversität (Berlin, Germany); MNHB, Museum of Natural History, Budapest; MWM, Museum of Thomas Witt (Munich, Germany); RYB, collection of R. Yakovlev (Barnaul, Russia); ZISP, Zoological Institute of Science Academy of Russian Federation (Saint-Petersburg, Russia).

\section{RESULTS}

\section{Annotated list of species}

Family Cossidae Leach, [1815]

Subfamily Catoptinae Yakovlev, 2009

Genus Catopta Staudinger, 1899

Dtsch. Entomol. Ztschr. Iris 12: 157-159. Type species: Catopta albimacula Staudinger, 1899.

1. Catopta albonubila (Graeser, 1888) (Figure 1) Cossus albonubilus Graeser, 1888, Berl. Entomol. Ztschr. 32: 119.

Type locality: Wlad. [Vladivostok, Primorskii Krai, southeast Russia].

Type material (holotype by monotypy) in ZISP.

General distribution: Russia (Chita, Primorje, Yakutia), central Mongolia, northeastern China, Korea.

Distribution in Mongolia: Central, Suhebaator and Khentei Aimaks. In Mongolia, ssp. argunica Yakovlev, 2007 (type locality: ezast Transbaicalia, Kuenga, 45 km SW of Sretensk [Russia, south part of Chita Region]; type material: holotype by original designation in MWM).

Material examined: 1 male, Mongolia, Suhebator Aimak, $25 \mathrm{~km} \mathrm{~N}$ of Bajanterem, $950 \mathrm{~m}$ above sea level (a.s.l.), 1.08.1965 (MWM); 2 males, Aimak Hentiy, Tsenher Mandal Modoto, 47 $50^{\prime}$ N, $109^{\circ} 05^{\prime}$ E, 12.7.1984, 1,700 $\mathrm{m}$ a.s.l., leg. Z. Weidenhoffer (MWM); 4 males, Chentai Aimak, Tsenkermandal, Modoto, 1,600-1,800 m a.s.l., $47^{\circ} 48^{\prime} \mathrm{N}, 109^{\circ} 04^{\prime} \mathrm{E}, 14.4 .1984$, leg. Cerny (MWM); 1 male, Central Aimak, Tuul Valley, Nalaikha, 1,600-1,800 m a.s.l., 6-8.7.1984, leg. Cornj (MWM); 1 male, Central Aimak, Bogdo ul Mountains, Zaisan Ulan Bator, 1,400-1,600 m a.s.l., $47^{\circ} 52^{\prime} \mathrm{N}, 106^{\circ} 55^{\prime} \mathrm{E}, 22.7 .84$, leg. Cerny (MWM). 
2. Catopta perunovi Yakovlev, 2007 (Figure 2)

Eversmannia 9: 13-14, pl. 2: 5-9, figs. 3-6, map 2.

Type locality: W. Altai Mountains, Ongudai [Russia, Altai Rep., Ongudai].

Type material (holotype by original designation) in MWM.

General distribution: Altai-Sayan Mountains, northwest Mongolia (Bayan-Ulgii, Uvs, Khovsgol district), Central Yakutia (Elanskoe, $60 \mathrm{~km}$ southwest of Pokrovsk).

Distribution in Mongolia: Khovd, Khubsugul and Bayan-Ulegei Aimaks.

Material examined: 1 male, Mongolia, Chovsgol Aimak, 4 km NW von der Stadt Moron, 1,500 m a.s.l., 19.07.1968, exp. Dr. Kaszab (MWM); 1 male, Mongolia, Chovsgol Aimak, $8 \mathrm{~km}$ N of somon Buren-Chaan, Delger Moron, 1,450 m a.s.l., 16.07.1968, exp. Dr. Kaszab (MWM), 4 males, W. Mongolia, Bayan-Ulegei Aimak, Kobdo-gol Valley, $20 \mathrm{~km}$ SW of Tsengel, 1,800 m, 26-30.07.2009, leg. E. Guskova and R.V. Yakovlev (RYB).

3. Catopta saldaitisi Yakovlev, 2007 (Figure 3)

Eversmannia 9: 15-16, pl. 2: 10-11, figs. 7-8, map 3.

Typelocality: Omnogovi Aimak, GoviAltai Mountains, Gurvan-Sayhan, Valley Yulin [southern Mongolia].

Type material (holotype by original designation) in MWM.

General distribution: Gobi Altai (Gurvan-Saihan) Mountains and southern part of Mongolian Altai (southern Mongolia: South-Gobi, Gobi-Altai and Hovd Aimaks).

Material examined: holotype, male, Omnogovi Aimak, Govi Altay Mountains, Gurvan Sayhan, Valley Yulin, 2,050 m, 43⒉ $\mathrm{N}, 104^{\circ} \mathrm{O} 3^{\prime} \mathrm{E}, 2^{2}-28.07 .1988$, leg. G. Szabwky (MWM); paratypes: 1 male, Daladzadgat, 11.7.81, leg. A. Weiss (MWM); 35 males, 2 females, Omnogovi Aimak, Govi Altay Mountains, Gurvan Sayhan, Valley Yulin, 2,050 $\mathrm{m}$ a.s.l., $43^{\circ} 27^{\prime} \mathrm{N}, 104^{\circ} \mathrm{O} 3^{\prime} \mathrm{E}, 25-28.07 .1988$, leg. G. Szabwky (MWM); 1 male, 1 female, S. Mongolia, Omnegov Aimak, Gurvan-Saikhan Mountains, $12 \mathrm{~km}$ NW of Tzokhor village, 2,300 $\mathrm{m}$ a.s.1., 14-15.07.2002, leg. S. Churkin (RYB); 2 male, 2 females, Mongolia, Gobi Altay Aimak, S. of Mongolian Altay, Alag Kharkhan Mountains, 2,500 $\mathrm{m}$ a.s.1., 5-7.07.2004. leg. Saldaitis, $45^{\circ} 31^{\prime} \mathrm{N}, 94^{\circ} \mathrm{O} 4^{\prime}$ E; 6 males, 1 female, Mongolia, Gobi Altay Aimak, Han Tayshir Mountains, Jargalan somon env., 2,050 $\mathrm{m}$ a.s.l., 12-14.07.2004, leg. Saldaitis, $46^{\circ} 50^{\prime} \mathrm{N}, 095^{\circ} 54^{\prime} \mathrm{E}$ (ASL); 1 male, Mongolia, Hovd Aimak, Sutay Uul Mountains, NW slopes, 1,900 m., 10.07.2004. leg. Saldaitis, $46^{\circ} 47^{\prime} \mathrm{N}$, $093^{\circ} 23^{\prime}$ N (ASL); 1 male, Mongolia, Hovd Aimak, Sutay Uul Mountains, NW slopes 2,000 m a.s.1., 11.07.2004, leg. Saldaitis, $46^{\circ} 49^{\prime} \mathrm{N}$, $093^{\circ} 18^{\prime} \mathrm{E}$ (ASL); 1 male, Mongolia, Gobi Altay Aimak, S of Mongolian Altay, Mogoin gol River, 1,920 m a.s.1., 8-9.07.2004. leg. Saldaitis, $45^{\circ} 39^{\prime} \mathrm{N}, 093^{\circ} 47^{\prime} \mathrm{E}$ (ASL); 1 male, SW Mongolia, Gobi-Altai Aimak, Mongolian Altai Mountains, S. slope, Mogoijn-Gol Valley, 11.07.2009, 1,80o m a.s.l., leg. R. Yakovlev and E. Guskova (RYB).
Genus Gobibatyr Yakovlev, 2004

Euroasian Entomol. J. 3(3): 217-218. Type species: Cossus colossus Staudinger, 1887.

4. Gobibatyr colossus (Staudinger, 1887) (Figure 4) Cossus colossus Staudinger, 1887, Stettin. ent. Ztschr. 48: 86.

Type locality: Kuldja-District [northwest China, Xinjiang, Kuldzha].

Type material (holotype by monotypy) in MHUB.

General distribution: Southeastern Kazakhstan, Kyrgyzstan, western Mongolia (Khovd Aimak, Arshantyn-Nuruu Mountains ), northwestern China.

Material examined: 1 female, Khovd Aimak, ArshantynNuruu Mountains, middle stream of Ulyastain Sala river, 2,100 m, 23.06.2004, leg. D. Djachenko (RYB).

5. Gobibatyr ustyuzhanini Yakovlev, 2004 (Figure 5)

Euroasian Entomol. J. 3(3): 218-219.

Type locality: Southern Mongolia, Gobi-Altai Aimak, $30 \mathrm{~km} \mathrm{~N}$ of Biger.

Type material (holotype by original designation) in ZISP.

General distribution: Mongolia (South-Gobi, Khovd and Gobi-Altai aimakAimaks).

Material examined: Holotype. Male. Southwestern Mongolia, Gobi-Altai Aimak, $30 \mathrm{~km} \mathrm{~N}$ of Biger, desert, 1100 m a.s.1., 27.06.1999, leg. Yakovlev R.V. (ZISP). Paratypes: 3 males, the same data, leg. V. Kovtunovitch, P. Ustyuzhanin and R. Yakovlev (RYB); 1 male, Southern Gobi Aimak, $70 \mathrm{~km}$ SW of Khan-Bogdo Somon, 18.06.1971, leg. Kerzhner (ZISP); 1 male, Southern Gobi Aimak, $50 \mathrm{~km}$ SSE of Noen, 1973, leg. G. Medvedev (ZISP); 1 male, Southern Gobi Aimak, Bulgan Somon, Talyn Bulay, 1,350 m a.s.1., 5.07.1967., exp. Z. Kaszab" (MWM); 4 males, 1 female, Chovd Aimak, Janatin Dolon, $40 \mathrm{~km}$ N Somon Manchan, SW bank of Char us nuur lake, 1,200 m a.s.l., 9.7.66., Z. Kaszab (MWM); 1 male, Chovd Aimak, $10 \mathrm{~km}$ SSW Somon Bulgan, 1,200 m a.s.1., 5.7.66., Z. Kaszab (MWM); 1 male, Gobi-Altai Aimak, between Beger nuur and somon Beger, 1,400 m a.s.1., 25.6.66., Z. Kaszab (MWM); 1 male, Gobi Altai Aimak, Baga nuur urd els, SE bank of Doroo nuur lake, 1,200 m a.s.1., 12.7.66. Z. Kaszab (MWM); 1 male, GobiAltai Aimak, Zachuj Gobi, $10 \mathrm{~km}$ N of Chatan chajrchan mountain, 1,750 m a.s.1., 27.06.66., Z. Kaszab (MWM). No type material: 4 males, 1 female, southwestern Mongolia, Hovd Aimak, Bodonchijn-Gol basin, HundijnGol river valley, 1,60o $\mathrm{m}$ a.s.1., 6.07.2009, leg. R. Yakovlev and E.Guskova (RYB); 1 male, southwestern Mongolia, Gobi-Altai Aimak, Mongolian Altai Mountains, S slope, Mogoijn-Gol Valley, 11.07.2009, 1,800 m a.s.l., leg. R. Yakovlev and E. Guskova (RYB).

Genus Acossus Dyar, 1905

Proc. U.S. Nat. Mus. 29: 178. Type species: Cossus undosus Lintner, 1878. 

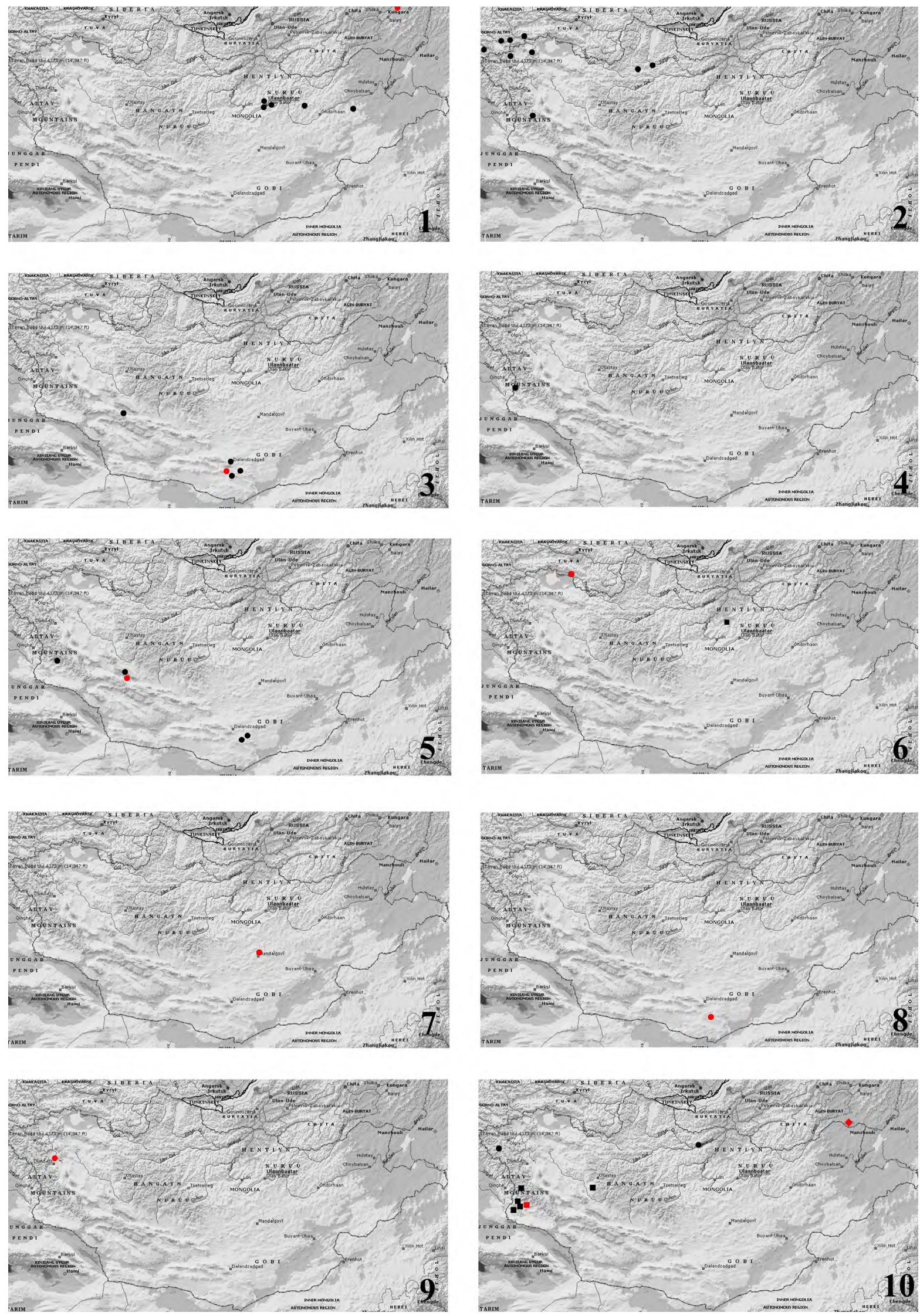

Figures 1-10. Distribution of Cossidae in Mongolia. 1: Catopta albonubila (Graeser, 1888). 2: Catopta perunovi Yakovlev, 2007. 3: Catopta saldaitisi Yakovlev, 2007. 4: Gobibatyr colossus (Staudinger, 1887). 5: Gobibatyr ustyuzhanini Yakovlev, 2004. 6: Acossus viktor (Yakovlev, 2004). 7: Chingizid gobiana (Daniel, 1970). 8: Chingizid transaltaica (Daniel, 1970). 9: Chingizid kosachevi Yakovlev, 2012. 10: Cossus cossus (Linnaeus, 1758); circles (•): ssp. cossus (Linnaeus, 1758); squares (ロ): ssp. deserta Daniel, 1969; diamonds (४): ssp. daurica Yakovlev, 2007. Red marks = type locality. 
Acossus viktor (Yakovlev, 2004) (Figure 6)

Lamellocossus viktor Yakovlev, 2004, Euroasian Entomol. J. 3(2): 157.

Type locality: Tuva, $30 \mathrm{~km} \mathrm{SW}$ of Samagaltai village, the Tes-Khem River valley [Russia, southern Siberia].

Type material (holotype by original designation) in ZISP.

General distribution: Russia, Southern Tuva (Erzin distr.). Probably in the Ubs Aimak of Mongolia.

\section{Genus Chingizid Yakovlev, 2011}

Neue Ent. Nachr. 66: 17. Type species: Lamellocossus transaltaica Daniel, 1970.

\section{Chingizid gobiana (Daniel, 1970) (Figure 7)}

Lamellocossus gobiana Daniel, 1970, Reichenbachia 13(19): 199-202

Type locality: Mittelgobi Aimak, $20 \mathrm{~km} \mathrm{~S}$ of Somon

Delgerzogt, [southern Mongolia].

Type material: holotype (by original designation) in MBNH.

General distribution: Mongolia (Middle-Gobi Aimak).

Material examined: holotype (male) and paratype (female) (NHMB, MWM).

7. Chingizid transaltaica (Daniel, 1970) (Figure 8)

Lamellocossus transaltaica Daniel, 1970, Reichenbachia 13(19): 203

Typelocality: SudgobiAimak, Gurban Sajchan ul Gebirge, zwischen Somon Churmen und Somon Bajandalaj, 24 km W von Churman, [southern Mongolia]

Type material: holotype (by original designation) in MBNH.

Distribution: Southern Mongolia

Distribution: South-Gobi Aimak (Transaltaian Gobi).

Material examined: holotype (male) and paratypes (2 females) (NHMB, MWM).

\section{Chingizid kosachevi Yakovlev, 2012 (Figure 9)}

Euroasian Ent. J. 11(6): 515.

Type locality: W. Mongolia, Hovd Aimak, near ErdeneBuren-Somon, Shurag-Gol Valley.

Type material (holotype by original designation) in ZISP.

General distribution: W. Mongolia (Great Lakes Valley).

Material examined: Holotype: male, W. Mongolia, Hovd Aimak, near Erdene-Buren-Somon, Shurag-Gol Valley, 1,400 m a.s.l., 13.06.2011, leg. R. Yakovlev (ZISP). Paratypes: 3 females, with the same data as holotype (RYB).

Genus Cossus Fabricius, 1793

Entomol. System. 3(2): 3. Type species: Phalaena cossus Linnaeus, 1758.

9. Cossus cossus (Linnaeus, 1758) (Figure 10)

Phalaena-Bombyx cossus Linnaeus, 1758, Systema Naturae: 504.

Type locality: Sweden.

Type material (syntypes) in LSL.
In Mongolia, two subspecies: Cossus cossus cossus (Linnaeus, 1758) and Cossus cossus deserta Daniel, 1969.

General distribution: all of the Palaearctic Region.

Distribution in Mongolia: ssp. cossus (L.) from northern part of Bayan-Ulegei Aimak (Kobdo-Gol Valley) to Selenge Aimak; ssp. deserta Daniel, 1969, from Khovd, Dzabkhan Aimaks. Probably in the eastern part of Mongolia: ssp. daurica Yakovlev, 2007.

Material examined: holotype of ssp. deserta (male) $10 \mathrm{~km}$ SSW of somon Bulgan, 1,200 m a.s.l., 4.7.1966, allotype and paratypes (2 males); paratypes (10 males) Somon Uench, Uench gol Valley, $2 \mathrm{~km} \mathrm{~N}$ of village, 1,450 $\mathrm{m}$ a.s.l., 7.7.1961 (MWM); 1 male, western Mongolia, Hovd Aimak, Dzhungarian Gobi, $15 \mathrm{~km}$ SW of Bulgan, 1,650 m a.s.l., 7.07.2003, R.V. Yakovlev (RYB); 3 males, 1 female, W. Mongolia, Hovd Aimak, near Erdene-BurenSomon, 1,400 m a.s.1., 4.07.2009, leg. R. Yakovlev and E. Guskova (RYB); 10 males, SW Mongolia, Hovd Aimak, Bodonchijn-Gol basin, Hundijn-Gol river Valley, 1,60o m a.s.1., 6.07.2009, leg. R. Yakovlev and E. Guskova (RYB). Subspecies cossus (L.): 3 males, W. Mongolia, BayanUlegei Aimak, Kobdo-gol Valley, $20 \mathrm{~km}$ SW of Tsengel, 1,800 $\mathrm{m}$ a.s.l., 26-30.07.2009, leg. E.V. Guskova and R.V. Yakovlev (RYB).

10. Cossus kerzhneri Yakovlev, 2011 (Figure 11)

Neue Entomol. Nachr. 66: 23.

Type locality: Mongolia, East-Gobi Aimak, 25 km SSW of Khuvsgel.

Type material (holotype) in ZISP.

General distribution: Mongolia (East-Gobi Aimak).

Material examined: holotype only.

Cossus shmakovi Yakovlev, 2004 (Figure 12)

Euroasian Ent. J. 3(2): 157.

Type locality: Tuva, $30 \mathrm{~km}$ SW of Samagaltai village, the Tes-Khem River valley.

Type material (holotype) in ZISP.

General distribution: Russia, southern Tuva (Erzin district); probably in Ubs Aimak of Mongolia.

Genus Holcocerus Staudinger, 1884

Memoires sur les Lepidopteres 1: 139. Type species: Cossus (Holcocerus) nobilis Staudinger, 1884

11. Holcocerus holosericeus Staudinger, 1884 (Figure 13) Cossus (Holcocerus) holosericeus Staudinger, 1884, Rom. Mém. Lép. 1: 141.

Type locality: Askhabad [Ashkhabad, Turkmenistan].

Type material (lectotype) in MHUB.

General distribution: Kazakhstan, southern Mongolia (Bayan-Khongor Aimak, Orog-Nuur Lake), Kirgizia, Uzbekistan, Tadzhikistan, Turkmenia, northwestern China, Afghanistan, Iran.

Material examined: 1 male, valley of lake Orok-Nor, and $\mathrm{N}$ slope of Ikhe-Bogdo, 7-13.7.926, leg. P. Kozlov (ZISP). 

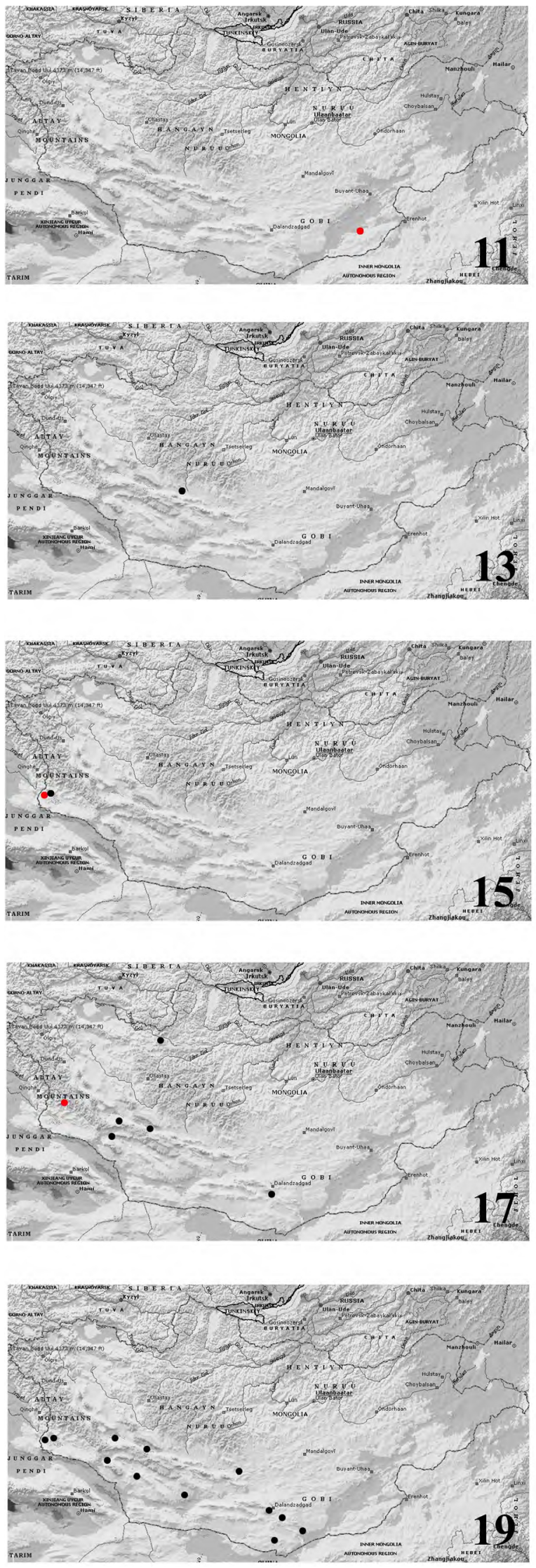
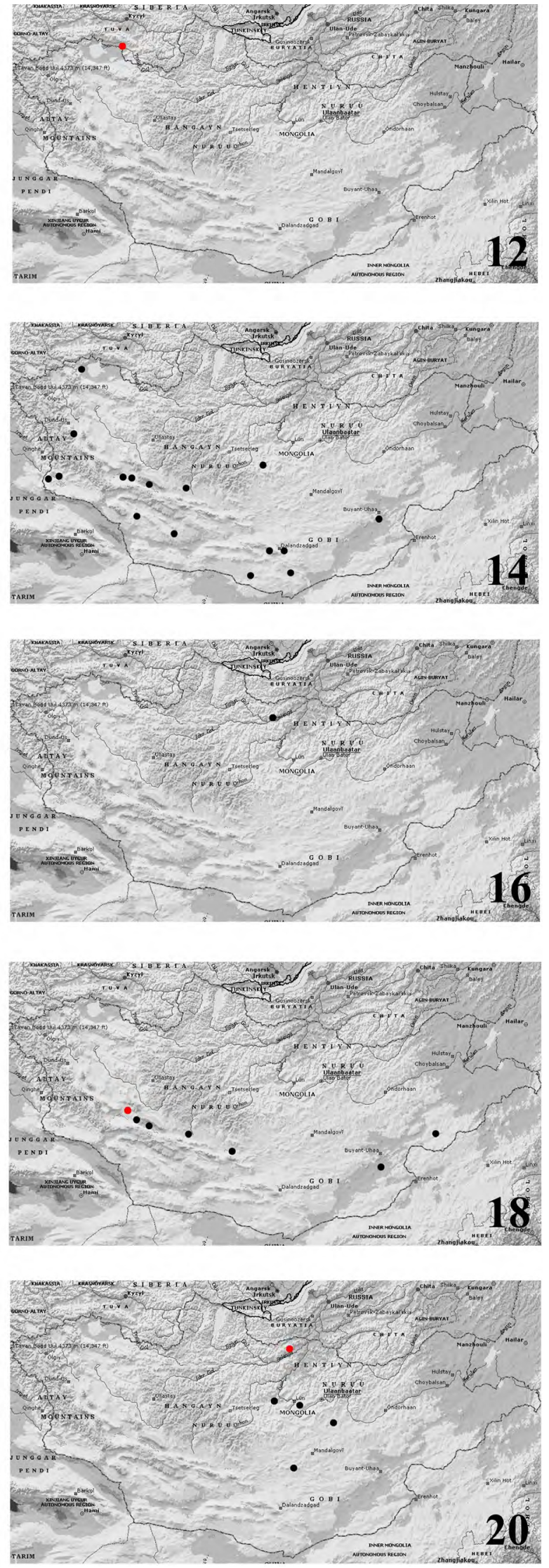

Figures 11-20. Distribution of Cossidae in Mongolia. 11: Cossus kerzhneri Yakovlev, 2011. 12: Cossus shmakovi Yakovlev, 2004. 13: Holcocerus holosericeus Staudinger, 1884. 14: Deserticossus consobrinus Püngeler, 1898. 15: Deserticossus beketi (Yakovlev, 2004). 16: Deserticossus tsingtauana (Bang-Haas, 1912). 17: Deserticossus churkini Yakovlev, 2006. 18: Deserticossus mongoliana (Daniel, 1969). 19: Barchaniella inspersa (Christoph, 1887). 20: Eogystia sibirica (Alpheraky, 1895). Red marks = type locality. 
Genus Deserticossus Yakovlev, 2006

Eversmannia Suppl. 1: 32. Type species: Cossus arenicola Staudinger, 1897.

12. Deserticossus consobrinus Püngeler, 1898 (Figure 14) Holcocerus consobrinus Püngeler, 1898, Soc. Entomol. 13: 57.

Type locality: Ili gebiet [Ili Valley, southeast Kazakhstan or northwest China].

Type material (holotype by original designation) in MHUB.

General distribution. Southern Siberia (Selenga Valley), Mongolia (Khovd, Gobi-Altai, Bayan-Khongor, Ubs, South-Gobi, East-Gobi Aimaks), Kazakhstan (including South Altai), Kirgizia, China (Xingjiang, Qinghai).

Material examined: 2 males, Gobi Altai Aimak, between Beger nuur lake and somon Beger, 1,400 m a.s.1., 25.6.66. (MWM); Mongol Els desert, $10 \mathrm{~km} \mathrm{SE}$ of Chechmort, 1,600 m a.s.1., 13.7.66; 1 male, Chovd Aimak, Jamatin Dolon, $40 \mathrm{~km}$ N of Somon Manchan, SW bank of Char us nur lake, 1,200 $\mathrm{m}$ a.s.1., 11.7.66 (MWM); 2 males, Sudgobi Aimak, Ostrand vom Zölöön ul Gebirge, $58 \mathrm{~km}$ WSW of Somon Bajandalaj, 1,500 m a.s.1., 16.6.67 (MWM); 1 male, Nojon Nuruu Range, Ovot Chuural, 1,500 m a.s.l., 20.6.67 (MWM); 1 male, somon Bulgan, Quelle talyn Bulag, $1350 \mathrm{~m}$ a.s.l., 5.7.67 (MWM); 1 male, $10 \mathrm{~km}$ NNE of Dalanzadgad, 1,450 m a.s.1., 7.7.67 (MWM); 2 males, Bajanchongor Aimak, Caganbulag in Cagan Bogd ul, 1,550 m a.s.l., 24.6.67 (MWM); 1 male, Uvs Aimak, Valley Chondlon gol, 32 $\mathrm{km} \mathrm{NW}$ of Ulangoom, 1,200 m a.s.l., 8.7.1968 (MWM); 3 males, 2 females, Bajan Hongor, Orog Lake env., 23-24.6.2003, $1230 \mathrm{~m}$ a.s.1., $45^{\circ} 05^{\prime} \mathrm{N}, 100^{\circ} 34^{\prime} \mathrm{E}$, leg. A. Saldaitis (MWM); 1 male, Bajanchongor Aimak, $60 \mathrm{~km}$ $\mathrm{S}$ of Bajanchongor, 27-30.06.03, 1,640 $\mathrm{m}$ a.s.1., $45^{\circ} 4 \mathrm{O}^{\prime} \mathrm{N}$, $100^{\circ} 41^{\prime} \mathrm{E}$, leg. A. Saldaitis" (MWM); 14 males, 1 female, Gobi Altai Aimak, 30 km N of Biger, desert, 23-27.06.1999, leg. V. Kovtunovitch, P. Ustyuzhanin and R. Yakovlev (RYB); 3 males, 1 female, Hovd Aimak, Dzhungarian Gobi, $45 \mathrm{~km}$ SW of Bulgan, Uvhod-Ula Mt., 1,350 m a.s.1., 8-10.07.2003, leg. V. Doroshkin, P. Ustyuzhanin, D. German and R. Yakovlev (RYB); 4 males, southwestern Mongolia, Gobi-Altai Aimak, Dzhungarian Gobi, AlagNuur lake, 16.07.2009, $1300 \mathrm{~m}$ a.s.l., leg. R. Yakovlev and E. Guskova (RYB); 1 male, Hovd Aimak, Dzhungarian Gobi, $15 \mathrm{~km}$ SW of Bulgan, 1,650 m a.s.l., 10.07.2003, leg. V. Doroshkin, P. Ustyuzhanin, D. German and R. Yakovlev (RYB); 1 female, Govi Altai Aimak, Zakhuin Gov', Khatan Khairhan Uul, 1,150 m a.s.1., 26.6.03, leg. S. Churkin (RYB); 3 males, 1 female, western Mongolia, Hovd Aimak, near Erdene-Buren-Somon, 1,400 m a.s.l., 4.07.2009, leg. R. Yakovlev and E. Guskova (RYB); 4 males, 1 female, southwestern Mongolia, Hovd Aimak, Bodonchijn-Gol basin, Hundijn-Gol riv. valley, 1,600 $\mathrm{m}$ a.s.1., 6.07. 2009, leg. R. Yakovlev and E. Guskova (RYB); 3 males, 2 females, Bajan Horog, Orog Lake env., 23-24.6.2003, 1,230 $\mathrm{m}$ a.s.l., $45^{\circ} 05^{\prime} \mathrm{N}, 100^{\circ} 34^{\prime} \mathrm{E}$, leg. A. Saldaitis (MWM); 1 male, Bajanchongor Aimak, $60 \mathrm{~km} \mathrm{~S}$ of Bajanchongor, 27-30.06.03, 1,640 m a.s.1., $45^{\circ} 40^{\prime} \mathrm{N}, 100^{\circ} 41^{\prime} \mathrm{E}$, leg. A. Saldaitis (MWM); 3 males, Bayankhongor, Ich-Bogd Mountains, 44 $59^{\prime} \mathrm{N}, 101^{\circ} 39^{\prime}$ E, 5.07.2000, leg. K. Špatenka (coll. W. Speidel, Bonn); 2 males, Gov'-Altaj, Sharga, Bayan-Gol, 46 ${ }^{\circ} 15^{\prime} \mathrm{N}, 095^{\circ} 19^{\prime}$ E, 17.07.2002, leg. K. Špatenka and Z. Weidenhoffer (coll. W. Speidel, Bonn).

13. Deserticossus beketi (Yakovlev, 2004) (Figure 15) Holcocerus beketi Yakovlev, 2004, Euroasian Ent. J. 3 (3): 221.

Type locality: Western Mongolia, Khovd Aimak, Dzhungarian Gobi, 45 km S of Bulgan-somon, Uvhod-Ula Mountain.

Type material (holotype by original designation) in ZISP.

General distribution: Mongolia, Khovd Aimak, Dzhungarian Gobi desert.

Material examined: only type series (holotype (male) and paratypes (7 males)).

\section{Deserticossus tsingtauana (Bang-Haas, 1912)}

(Figure 16)

Holcocerus tsingtauana Bang-Haas, 1912, D. ent. Z. Iris 26: 109.

Type locality: Tsingtau [China, Shandong province, Tsingtau Mountains].

Type material (syntypes) in MHUB and MWM.

General distribution: Russia (Primorskii Krai, Chita region, Buryatia Republic), Mongolia (Central Aimak), China (Heilongjiang, Jilin, Liaoning, Hebei, Shandong, Jiangsu, Shanxi, Shaanxi, Ningxia, Gansu, Inner Mongolia, Beijing, Tianjin, Shanghai, Anhui, Yunnan), Korea.

Material examined: 1 male, Mongolia, Central Aimak, $25 \mathrm{~km} \mathrm{~W}$ of Erdennesant, 14-15.06.2003, 47 22' N, $104^{\circ} 13^{\prime}$ E, 1,260 $\mathrm{m}$ a.s.l., leg. A. Saldaitis (MWM).

\section{Deserticossus churkini Yakovlev, 2006 (Figure 17)}

Eversmannia Supplement 1: 58-59.

Type locality: W. Mongolia, Hovd Aimak, middle stream of Uenchin-Gol river.

Type material (holotype by original designation) in MWM.

Distribution: Mongolia (Khovd, Bayan-Khongor, Ubs and South-Gobi Aimaks).

Material examined: holotype (male), W. Mongolia, Hovd Aimak, middle stream of Uenchin-Gol River, 1,750 $\mathrm{m}$ a.s.1., 2.07.2004, leg. R. Yakovlev and D. Ryzhkov (MWM), paratypes: 6 males, 2 females, Bayan-Khongor Aimak, $140 \mathrm{~km} \mathrm{~S}$ of Shine-Dzhinst, oasis Ekhin-Gol, 12-16.07.1981. leg. A. Lvovskii (ZISP), 1 male, South-Gobi Aimak, Dzemgin-Gobi, $25 \mathrm{~km}$ SSW of KhajlastynKhuduk, 20.06.971, leg. I. M. Kerzhner (ZISP), 1 male, Northern Gobi, Kholt, 10.08.1926, leg. P. Kozlov (ZISP); 1 female, Mongolia, Gobi-Altai Aimak, $30 \mathrm{~km}$ SE of Bugat, 2,030 $\mathrm{m}$ a.s.l., 10.06.2003, leg. A. Saldaitis (MWM). 
16. Deserticossus mongoliana (Daniel, 1969) (Figure 18) Holcocerus mongoliana Daniel, 1969a, Reichenbachia 11: 274-275.

Type locality: Mongolia, Gobi-Altai Aimak, Mongol Els, $10 \mathrm{~km} \mathrm{SE}$ of Chechmort.

Type material (holotype by original designation) in MNHB.

Distribution: Mongolia (Gobi-Altai, Bayan-Khongor, Ubs, South-Gobi, Sukhebaator, Uburkhangai and EastGobi Aimaks).

Material examined: holotype (male) Gobi Altai Aimak, Mongol Els, $10 \mathrm{~km} \mathrm{SE} \mathrm{Chechmort,} 1600 \mathrm{~m}$ a.s.1., 13.7.66 (MNHB); paratypes: 1 male, Gobi Altai Aimak, Baga nuuryn nurd els, SE bank of Doroo nuur lake, 1,200 $\mathrm{m}$ a.s.1., 12.7.66 (MWM); 1 male, 2 females, East-Gobi Aimak, Cagan Elis, $30 \mathrm{~km}$ ESE of Zuun-Bajan, $800 \mathrm{~m}$ a.s.1., 22.6.63 (MWM); 1 female, Bajanchongor Aimak, SE bank Orog nur lake, $1200 \mathrm{~m}$ a.s.l., 23.6.1964 (MWM); 1 male, Bajanchongor Aimak, $5 \mathrm{~km} \mathrm{~S} \mathrm{of} \mathrm{Somon}$ Bogd, $1200 \mathrm{~m}$ a.s.1., 24.6.64 (MWM); 1 male, Suchebator Aimak, Ongon Elis, $10 \mathrm{~km} \mathrm{~S}$ of Somon Chongor, 900 $\mathrm{m}$ a.s.1., 3.8.65 (MWM). Non-type material: 2 males, Uburchangai Aimak, $130 \mathrm{~km}$ ESE of somon Bajanleg, 1,150 m a.s.1., 3.7.67 (MWM) ; 1 male, Khovd Aimak, 30 $\mathrm{km} \mathrm{N}$ of Biger, 23-27.06.1999, leg. V. Kovtunovitch, P. Ustyuzhanin and R. Yakovlev (RYB); 4 males, 2 females, Bayankhongor, desert, $29 \mathrm{~km}$ of Khoriult, $45^{\circ} 38^{\prime} \mathrm{N}$; $100^{\circ} 38^{\prime}$ E, 8-9.07.2000, leg. K. Špatenka (coll. W. Speidel, Bonn); 2 males, Bayankhongor, river Tuy Gol, $46^{\circ} 43^{\prime} \mathrm{N}$; $100^{\circ} 55^{\prime}$ E, 29.06.2000, 2,360 m a.s.l., leg. K. Špatenka (coll. W. Speidel, Bonn); 1 male, East-Gobi Aimak, 2 $\mathrm{km} \mathrm{SW}$ of Mandakh, 1,300 m a.s.1., 5-7.2002., 44 ${ }^{\circ} 24^{\prime} \mathrm{N}$, $108^{\circ} 13^{\prime} \mathrm{E}$ (MWM); 1 male, Central Aimak, $20 \mathrm{~km} \mathrm{NW}$ of Bayan Tsadmani, Tsagan-Davaa, $1,500 \mathrm{~m}, 48^{\circ} 17^{\prime} \mathrm{N}$, 106ㅇํ' E; 18.07.1988, leg. Cs. Szaboky (MWM); 1 male, Omnogovi Aimak, Govi-Altai Mountains, GurvanSayhan, Valley Yulin, 2,050 m a.s.1., 43⒉ $7^{\prime} \mathrm{N}, 104^{\circ} 03^{\prime} \mathrm{E}$, 28.07.1988. leg. Cs. Szaboky (MWM); 1 male, East-Gobi Aimak, $7 \mathrm{~km}$ SW of Khatan-Bulak, $43^{\circ} 07^{\prime} \mathrm{N}, 109^{\circ} \mathrm{O} 3^{\prime}$ E, 9.08.2002, leg. Lind (MWM); 1 male, southern Gobi Aimak, Bordzon-Gobi, 8o km SSE of Nomgon somon, 5.08.967, leg. Zaitzev (ZISP).

\section{Genus Barchaniella Yakovlev, 2006}

Eversmannia Supplement 1: 75. Type species: Holcocerus inspersus Christoph, 1887.

17. Barchaniella inspersa (Christoph, 1887) (Figure 19) Holcocerus inspersus Christoph, 1887b, Stett. Entomol. Ztschr. 48: 163.

Typelocality:Ashkhabad [Turkmenistan, Ashkhabad]. Type material (lectotype) in ZISP.

General distribution: Kazakhstan, Uzbekistan, Turkmenia, southwestern China (Xinjang), southwestern Mongolia (Khovd, Gobi-Altai, Bayn-Khongor, Uburkhangai, South-Gobi and East-Gobi Aimaks).

Material examined: 3 males, 6 females, Hovd Aimak, Dzhungarien Gobi, $45 \mathrm{~km}$ SW of Bulgan, Uvhod-Ula
Mountain, 1,350 m a.s.1., 8-10.07.2003., V. Doroshkin, P. Ustyuzhanin, D. German and R. Yakovlev (RYB); 5 males, 4 females, Govi Altai Aimak, Zakhuin Gov', Khatan Khairhan Uul, 1,150 $\mathrm{m}$ a.s.1., 26.6.03, leg. S. Churkin (RYB); 1 male, Gobi-Altai Aimak, $5 \mathrm{~km}$ S of pass between Adzh-Bogd uul and Tschijn Schar nuruu Ranges, 1,600 m a.s.1., Exp. Dr. Kaszab, 29.06.1966 (MWM); 1 female, Gobi Altai Aimak, Zachuj Gobi, $10 \mathrm{~km}$ of Chatan chairhan Range, 1,150 m a.s.l., 27.6.66 (MWM); 2 males, Hovd Aimak, $60 \mathrm{~km}$ W of Bulgan, 1,120 m a.s.1., 20.06.1964, exp. Dr. Kaszab (MWM); 3 males, 10 km SSW of Bulgan, 1,200 m a.s.1., 5.07.1966 (MWM); 2 males, Bajanchongor Aimak, Talyn Bilgech bulag, between Tost ul and Cagan Bogd ul Ranges, $47 \mathrm{~km}$ E of Caganbulag, 1,200 m a.s.l., 23.6.67 (MWM); 2 males, South-Gobi Aimak, SW bank of Dund gol lake, 1,300 m a.s.l., 18.6.67 (MWM); 14 males, SW Mongolia, Gobi-Altai Aimak, Dzhungarian Gobi, Alag-Nuur lake, 16.07.2009, 1,300 m a.s.l., leg. R. Yakovlev and E. Guskova (RYB).

Genus Eogystia Schoorl, 1990

Zool. Verhandelingen 263: 71-72. Type species: Hypopta sibirica Alpheraky, 1895.

\section{Eogystia sibirica (Alpheraky, 1895) (Figure 20)}

Hypopta sibirica Alpheraky, 1895, Dt. Entomol. Ztschr. Iris 8: 185-186.

Type locality: Barabash [southeas Russia, Primorskii krai, Barabash-Levada]

Type material (lectotype) in ZISP.

General distribution: Russia (southern part of Chitinskaya and Amurskaya Oblast' [Dauria], Primorje), northern and central Mongolia (Central, Bulgan and Domod Aimaks), China (Jilin, Inner Mongolia, Hebei, Shandong).

In Mongolia, ssp. Eogystia sibirica krusheki Yakovlev, 2007.

Reported by Daniel from: Bulgan Aimak, $5 \mathrm{~km}$ W of Somon Daschintshilen, 1,140 m a.s.1., 2.7.64; $26 \mathrm{~km}$ E of Somon Lun, 1,180 m a.s.1., 3.7.64 (Daniel, 1965); Bulgan Aimak, $20 \mathrm{~km} \mathrm{~W}$ of Somon Bajannuur $(220 \mathrm{~km} \mathrm{~W}$ of Ulan-Baator), 1,100 $\mathrm{m}$ a.s.1., 17.6.1966 [Daniel, 1969]; Central Aimak, $12 \mathrm{~km}$ S of Somon Bajanbaraat, 1,380 m a.s.1., 8.6.67; Central-Gobi Aimak, Choot bulag, between Somon Chuld and Somon Delgerchangaj, $38 \mathrm{~km}$ ENE of Delgerchangaj, 1,480 m a.s.l., 10.06.1967; Bajanchongor Aimak, Zinst ul Range, $50 \mathrm{~km}$ E of Somon Schine zinst, 2,000 m a.s.l., 30.06.1967 (Daniel, 1970); Bulgan Aimak, $11 \mathrm{~km}$ W of Somon Bajannuur, southern bank of Bajan nuur lake, 1,00o m a.s.l., 14.06.1968 (Daniel 1973).

19. Eogystia kaszabi (Daniel, 1965) (Figure 21)

Isoceras kaszabi Daniel, 1965, Reichenbachia 7 (10): 100-102.

Type locality: Mongolia, Bayan-Hongor Aimak, SE bank of Orog nur lake.

Type material (holotype by original designation) in MNHB. 
General distribution: southern Mongolia (BayanKhongor Aimak), China (Shaanxi, Ningxia, Qinghai, Inner Mongolia).

Material examined: holotype (male) and paratype (male), Bayan-Hongor Aimak: SO bank of Orog nur lake, 1,200 $\mathrm{m}$ a.s.1., 23.6.64 (MNHB and MWM); paratypes ( 3 males), $8 \mathrm{~km}$ of Somon Zinst, 1,400 m a.s.l., 25.6.64 (3 paratypes in MWM). Non-type material: 4 males, Mongolia, Bajanchongor Aimak, $60 \mathrm{~km} \mathrm{~S}$ of Bajanchongor, 27-30.06.03, 1,640m a.s.l., 45 40' N, $100^{\circ} 41^{\prime} \mathrm{E}$, leg. A. Saldaitis (MWM).

Genus Kerzhnerocossus Yakovlev, 2011

Neue Ent. Nachr. 66: 37. Type species: Kerzhnerocossus sambainu Yakovlev, 2011.

20. Kerzhnerocossus sambainu Yakovlev, 2011 (Figure 22) Neue Ent. Nachr. 66: 38.

Type locality: East Aimak, from Modon-Obo Mountain to Tamsag-Bulak.

Type material (holotype) in ZISP.

General distribution: Mongolia (East Aimak).

Material examined: holotype and 2 paratypes (all males), Mongolia, East Aimak, from Modon-Obo Mountain to
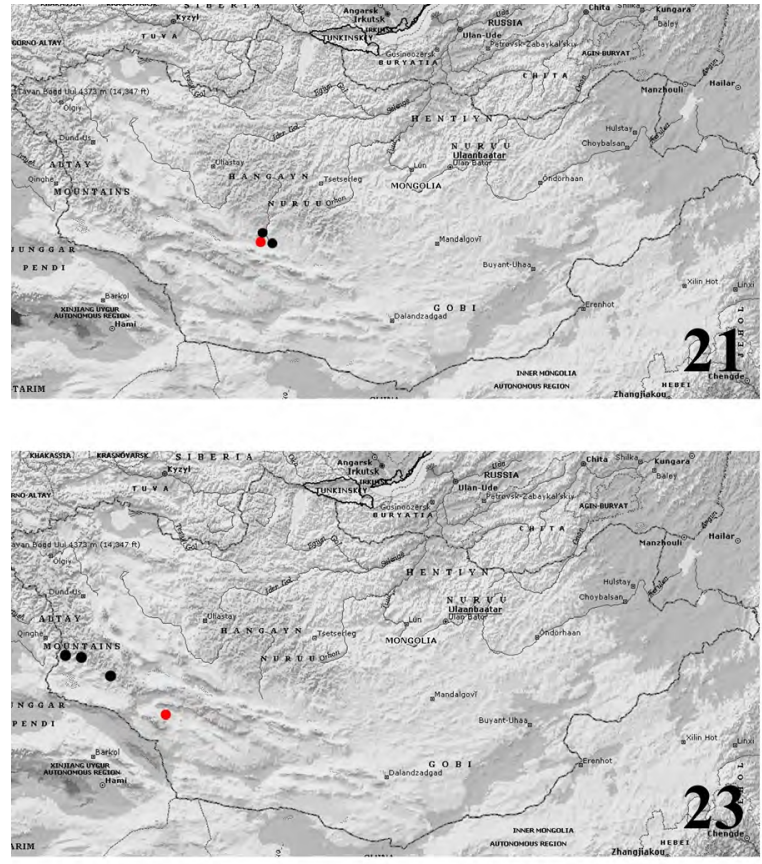

Tamsag-Bulak, 20.06.976, leg. Kerzhner (ZISP).

Genus Dyspessa Hübner, [1820]

Verz. bekannter Schmett.: 194. Type species: Phalena pantherina Hübner, 1790.

21. Dyspessa saldaitisi Yakovlev, 2011 (Figure 23)

Neue Ent. Nachr. 66: 45.

Type locality: Mongolia, Gobi-Altai Aimak, south of Mongolian Altai, Mogoin-Gol River.

Type material (holotype) in MWM.

General distribution: Mongolia (Khovd and GobiAltai Aimaks).

Material examined: holotype and 3 paratypes (all males) Mongolia, Gobi-Altai Aimak, south of Mongolian Altai, Mogoin-Gol River, 1,920 m a.s.l., 15-18.06.2004, leg. Saldaitis (MWM); 1 paratype (male), $1 \mathrm{~m}$, SW Mongolia, Gobi-Altai Aimak, Mongolian Altai Mountains (southern slope), Mogoijn-Gol Valley, 6-8.07.2010, $1800 \mathrm{~m}$ a.s.l., $45^{\circ} 39^{\prime} \mathrm{N}$, $093^{\circ} 47^{\prime} \mathrm{E}$, leg. R. Yakovlev and E. Guskova (RYB). No type material: 3 males, southwestern Mongolia, Hovd Aimak, Bodonchijn-Gol basin, Valley Hundijn-Gol River valley, 1,600 $\mathrm{m}$ a.s.l., $46^{\circ} 06^{\prime}$ N, $092^{\circ} 30^{\prime}$ E; 28.05.2011, leg. R. Yakovlev (RYB);
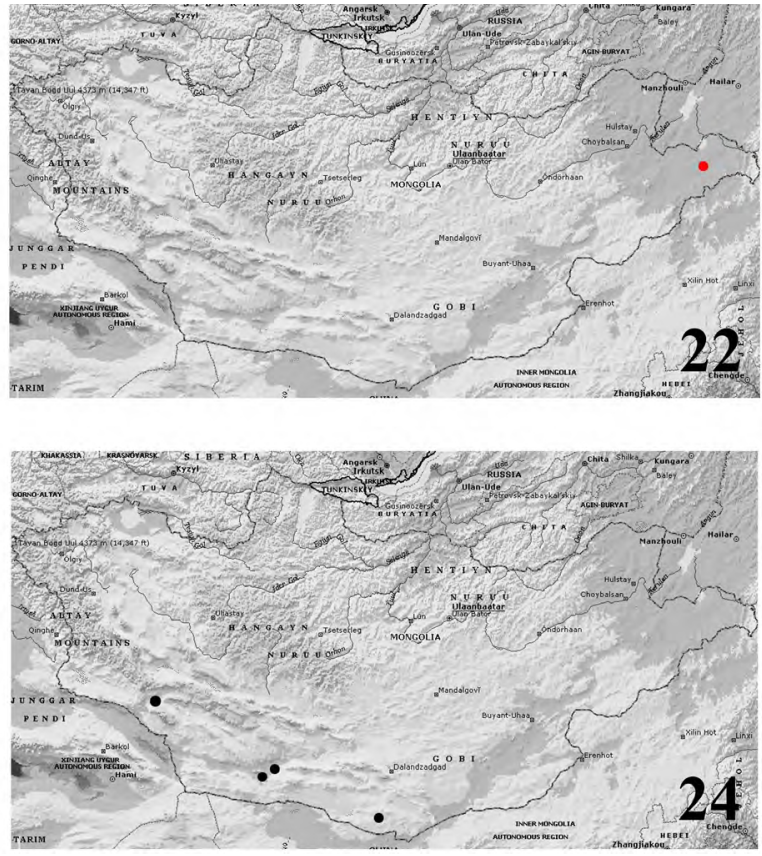

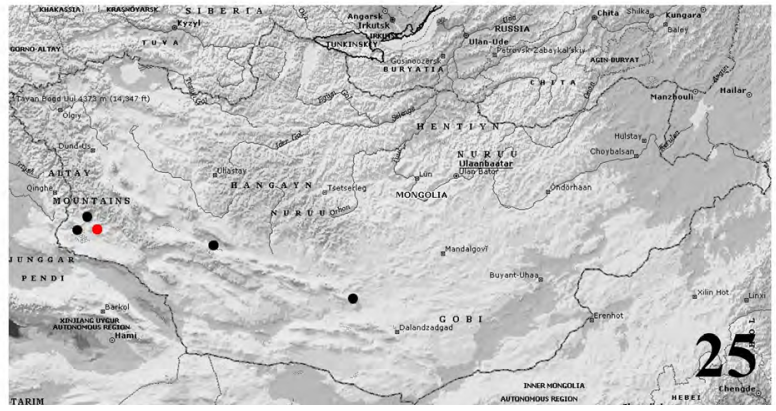

Figures 11-20. Distribution of Cossidae in Mongolia. 21: Eogystia kaszabi (Daniel, 1965). 22: Kerzhnerocossus sambainu Yakovlev, 2011. 23: Dyspessa saldaitisi Yakovlev, 2011. 24: Cecryphallus nubila (Staudinger, 1895). 25: Phragmataecia anikini Yakovlev, 2011. Red marks = type locality. 
4 males, W Mongolia, Hovd Aimak, near of AltanSoembo, Uvchugijn-Serven Mountain, 1,700 m a.s.l., $45^{\circ} 39^{\prime} \mathrm{N}, 093^{\circ} 21^{\prime}$ E; 29.05.2011, leg. R. Yakovlev (RYB); 1 male, Western Mongolia, Gobi-Altai Aimak, 15 km SEE of Altan-Soembo, Hundlengijn-Shil place, 1,650 m a.s.l., $45^{\circ} 37^{\prime} \mathrm{N}$, 093 $31^{\prime}$ E, 30.05.2011, leg. R. Yakovlev (RYB); 2 males, SW Mongolia, Gobi-Altai Aimak, Dzhungarian Gobi desert, Alag-Nuur lake (near Ajlyn-Tsagan-

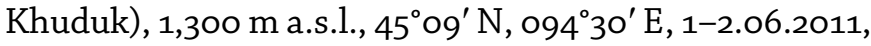
leg. R. Yakovlev (RYB); 2 males, southwestern Mongolia, Hovd Aimak, $30 \mathrm{~km} \mathrm{~S}$ of Altai somon, Bodonchijn-Gol river valley (under stream), Elkhony-Ekhen-Tal place,

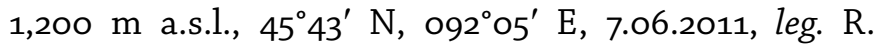
Yakovlev (RYB); 2 males, W Mongolia, Hovd Aimak, Bulgan-gol basin, Bayan-Gol river valley, ArshantynNuruu Mountains, Ulyastajn-Sala river, 1,900 m a.s.l., $46^{\circ} 21^{\prime}$ N; 091 o8' E, 9-10.06.2011, leg. R. Yakovlev (RYB).

\section{Subfamily Zeuzerinae Boisduval, [1828]}

\section{Genus Cecryphalus Schoorl, 1990}

Zool. Verhandelingen 263: 156-157. Type species: Zeuzera nubila Staudinger, 1895 .

22. Cecryphallus nubila (Staudinger, 1895) (Figure 24). Zeuzera nubila Staudinger, 1895, Dtsch. Entomol. Ztschr. Iris 8: 341.

Type locality: Kaschgar [northwestern China, Tura].

Type material (cotypes) in MHUB.

General distribution: Southern Kazakhstan, Kirgiziya, southern Mongolia (Gobi-Altai, Bayan-Khongor and South-Gobi Aimaks), Uzbekistan, Tadzhikistan, Azerbaijan, southern Armenia, Turkmenistan, northern Iran, and Xinjiang, China.

Reported by Daniel from South-Gobi Aimak, $100 \mathrm{~km}$ W of Ovot Chuural, $22 \mathrm{~km} \mathrm{~W}$ of Sajryn chudag, 1,250 $\mathrm{m}$ a.s.1., 22.6.1967; Bajanchongor Aimak, Caganbulag, Cagan Bogd ul Range, 1,550 m a.s.l., 24.06.1967; Oasis Echin gol, $90 \mathrm{~km} \mathrm{NE}$ of Caganbulag, $950 \mathrm{~m}$ a.s.l., 27-28.6.1967 (Daniel, 1970).

Material examined: 2 males, SW Mongolia, Gobi-Altai Aimak, Dzhungarian Gobi, Alag-Nuur lake, 9-10.07.2010, 1,300 m a.s.l., 45 o9' N; $94^{\circ} 30^{\prime}$ E; leg. R. Yakovlev, E. Guskova (RYB).

Genus Phragmataecia Newman, 1850

Newman, 1850, Zoologist 8: 2931. Type species: Noctua arundinis Hübner, [1808]

23. Phragmataecia anikini Yakovlev, 2011 (Figure 25).

Neue Ent. Nachr. 66: 53.

Type locality: Chovd Aimak, $3 \mathrm{~km} \mathrm{~N}$ von Somon Uench, im Tal des Flusses Uench gol.

Type material (holotype) in MWM.

General distribution: Southwestern Mongolia (Khovd and Gobi-Altai Aimaks).

Material examined: holotype (male) Mongolia, Chovd
Aimak, $3 \mathrm{~km} \mathrm{~N}$ of Somon Uench, valley of Uench gol, 1,450 $\mathrm{m}$ a.s.1., 7.7.1966; paratypes: 4 males, same data (MWM); 2 males, Mongolia, $10 \mathrm{~km}$ SSW of Somon Bulgan, 1,200 m a.s.1., 4.7.1966 (MWM); 2 males, Mongolia, Uburchangai Aimak, Oasis Chacar-usni chudag, $100 \mathrm{~km}$ ESE of Somon Bajanleg, 1,200 m a.s.1., 3.7.1967; 1 male, Mongolia, Hovd Aimak, $13 \mathrm{~km} \mathrm{~S}$. of Altai Somon centre along the river Bodonch, 1,300 $\mathrm{m}$ a.s.1., $45^{\circ} 57^{\prime} \mathrm{N}$, $092^{\circ} 50^{\prime} \mathrm{E}, 19.5 .1990$, leg. G. Fabian, M. Hreblay, I. Peregovits and G. Roncay (MWM); 3 males, Mongolia, Gobi-Altai Aimak, 10-30 $\mathrm{km} \mathrm{N}$ of Biger, 22-23.06.1999, leg. V. Kovtunovich, P. Ustjuzhanin and R. Yakovlev (RYB); 5 males, Mongolia, Hovd Aimak, Barun-Khurai desert, $15 \mathrm{~km}$ S [of] Bulgan, 10.07.2007, leg. E. Gus'kova, V. Anikin and R. Yakovlev (RYB, ZISP).

\section{DISCUSSION}

The fauna of Cossidae of Mongolia comprises 23 species (or very likely 25 species, if including species described from the adjacent region of southern TuvaAcossus viktor (Yakovlev, 2004) and Cossus shmakovi Yakovlev, 2004). These belong to 12 genera (or 13, including Acossus Dyar, 1905). It is important to note that the cossid fauna of Mongolia is highly specific which is confirmed by the presence of 13 endemic species (Catopta saldaitisi, Gobibatyr ustyuzhanini, Chingizid gobiana, Ch. transaltaica, Ch. kosachevi, Cossus kerzhneri, Deserticossus beketi, D. churkini, D. mongoliana, Eogystia kaszabi, Kerzhnerocossus sambainu, Dyspessa saldaitisi and Phragmataecia anikini) and two endemic genera (Kerzhnerocossus and Chingizid) All Mongolian endemics, except for Catopta saldaitisi Yakovlev, 2007, inhabit deserts and semideserts. The high percentage of endemics (more than $50 \%$ of the species and $10 \%$ of the genera), along with the overall depleted nature of the fauna, is evidence of its extremely ancient derivation from the Gobian fauna. The high endemism in Mongolia of the Cossidae is unique over all of the Palaearctic studied by me except in Yunnan, China. Available data suggests that the deserts of Mongolia are one of the centers of speciation of Cossidae in Eurasia.

\section{ACKNOWLEDGEMENTS}

I wish to express my deepest gratitude to all those who helped me study Mongolian Cossidae. Firstly, I thank Thomas Witt for access to his collection, including type specimens, and his library; curators of museum collections, S. Sinev, V. Mey, A. Hausmann, W. Speidel, and L. Ronkay for access to their collections; and entomologists A. Saldaitis, S. Churkin, and M. Ströhle for material from Mongolia and adjacent regions given for study. My colleagues offered great support to me during expeditions to remote parts of Mongolian Altai: S.V. Smirnov, V.V. Anikin, E.V. Guskova, P. Ya. Ustyuzhanin, V.N. Kovtunovich, D.A. German, P.A. Kosachev, V.V. 
Doroshkin, and D.V. Ryzhkov. The expedition by AltSU would not be possible without selfless labour of our driver, M. Sidorov. A large help in organization of studies were rendered by A.I. Shmakov, U. Beket, and G. Habiev. I thank V.V. Zolotuhin for important advice, constructive critical notes and comprehensive help.

\section{LITERATURE CITED}

Daniel, F. 1965. 53. Bombyces et Sphinges. 1. Ergebnisse der zoologische Forschungen von Dr. Z. Kaszab in der Mongolei. Reichenbachia 7(10): 99-102.

Daniel, F. 1969. 165. Bombyces et Sphinges. 3. Ergebnisse der zoologische Forschungen von Dr. Z. Kaszab in der Mongolei. Reichenbachia 11(25): 274-277.

Daniel, F. 1970. 200. Bombyces et Sphinges. 4. Ergebnisse der zoologische Forschungen von Dr. Z. Kaszab in der Mongolei. Reichenbachia 13(19): 199-203.

Daniel, F. 1973. 262. Bombyces et Sphinges. 5. Ergebnisse der zoologische Forschungen von Dr. Z. Kaszab in der Mongolei. Faunistische Abhandlungen 4(19): 168-169.

Nieukerken, van E.J., L. Kaila, I.J. Kitching, N.P. Kristensen, D.C. Lees, J. Minet, C. Mitter, M. Mutanen, J.C. Regier, T.J. Simonsen, N. Wahlberg, S.-H. Yen, R. Zahiri, D. Adamski, J. Baixeras, D. Bartsch, B.Å. Bengtsson, J.W. Brown, S.R. Bucheli, D.R. Davis, J. De Prins, W. De Prins, M.E. Epstein, P. Gentili-Poole, C. Gielis, P. Hättenschwiler, A. Hausmann, J.D. Holloway, A. Kallies, O.
Karsholt, A. Kawahara, J.C. Koster, M.V. Kozlov, J.D. Lafontaine, G. Lamas, J.-F. Landry, S. Lee, M. Nuss, K.-T. Park, C. Penz, J. Rota, B.C. Schmidt, A. Schintlmeister, J.C. Sohn, M.A. Solis, G.M. Tarmann, A.D. Warren, S. Weller, R.V. Yakovlev, V.V. Zolotuhin and A. Zwick. 2011. Order Lepidoptera Linnaeus, 1758. Zootaxa 3148: 212-221.

Yakovlev, R.V. 2004. Carpenter-moths (Lepidopetera, Cossidae) of Mongolia. Euroasian Entomological Journal 3(3): 217-224.

Yakovlev, R.V. 2006. A revision of carpenter moths of the genus Holcocerus Staudinger, 1884 (s. 1.). Eversmannia Supplement 1: 104 pp. [In Russian].

Yakovlev, R.V. 2007a. Cossidae (Lepidoptera) of Russia. Eversmannia 9: 11-33. [in Russian].

Yakovlev, R.V. 2009. Systematic review of the Goat Moth-Cossus cossus (Linnaeus, 1758) (Lepidoptera, Cossidae). Amurian Zoological Journal 1(1): 58-71. [In Russian].

Yakovlev, R.V. 2011. Catalogue of the family Cossidae of the Old World (Lepidoptera). Neue Entomologische Nachrichten 66: 1-130.

Yakovlev, R.V. 2012. A review of the genus Chingizid Yakovlev, 2011 (Lepidoptera, Cossidae) with description of new species. Euroasian Entomological Journal 11(6): 513-516 (in Russian).

Yakovlev, R.V. and V.V. Doroshkin. 2004. New data of Macrolepidoptera for the fauna of Mongolia. II. Atalanta 35(3/4): 390-398.

Received: 31 July 2013

Accepted: 19 August 2015

Academic editor: Cristiano A. Iserhard 